\title{
SGLT-2i and Cardiovascular Outcomes: A Meta-Analysis of The Asian Cohort
}

\author{
Samit Ghosal $^{1^{*}}$, Binayak Sinha ${ }^{2}$ \\ ${ }^{1}$ Consultant Endocrinologist, Nightingale Hospital, Kolkata, India \\ ${ }^{2}$ Consultant Endocrinologist, AMRI Hospitals, Kolkata, India
}

Received: December 15,2018; Accepted: December 26,2018; Published: December 29, 2018

*Corresponding author : Samit Ghosal, Consultant Endocrinologist, Nightingale Hospital, Kolkata, India, Tel: +91-9674328281; Email: ramdasghosal@ gmail.com

\section{Letter}

Sir, Recent diabetes guidelines have upgraded the position of sodium glucose cotransporter 2 inhibitors (SGLT-2i) based on the publication of cardiovascular outcomes trials (CVOT). However, divergent signals have emerged from the 3 CVOTs. It is speculated that these differences were due to differing patient population and cardiovascular (CV) risk status, suggesting that the benefits of SGLT-2i were driven primarily by their effect on the pump (hospitalization for heart failure (hHF) and CV death) and not on the pipe (myocardial infarction, stroke \& peripheral arterial disease). [1]

However, another important question which arises from all these trials, is whether the $\mathrm{CV}$ benefits extended to the Asian subset, a cohort known to have worse heart disease yet grossly under-represented in most of the CVOTs. In the absence of a planned RCT in the Asian cohort, conducting a pooled metaanalysis seems to be the next best option.

We conducted a meta-analysis on 4,997 Asian patients included in the 3 CVOTs with SGLT-2i. The aim was to look at the impact of SGLT-2i on the pump \& the pipe. Since we did not deal with patients, ethical committee approval was not sought. Initial database search was conducted in Cochrane library using the terms "sodium glucose cotransporter 2 inhibitor", "dapagliflozin", "empagliflozin", "canagliflozin", "ertugliflozin" and "MACE". Of the 16 citations retrieved from the initial search, the studies for final analysis (3) were based on a pre-specified inclusion criterion (randomized controlled trial, \& more than 1000 patients followed up for at least 12 months) [2-4]. This meta-analysis was conducted using the comprehensive meta-analysis (CMA) software version 3, Biostat Inc., Englewood, NJ, United States. Heterogeneity was assessed using the Cochrane Q and Higgins's I2 test and publication bias was assessed by funnel plot.

The hazard ratio (HR) for MACE in the pooled Asian cohort was $0.84(95 \% \mathrm{CI}: 0.67-1.05, \mathrm{P}=0.13)$, which was statistically non-significant. However, the "CV death or hHF" composite was significantly lowered by $25 \%$ (95\% CI: $0.60-0.93, \mathrm{P}=0.01)$. (Fig 1)

This finding contrasts with the data from the overall cohort, where both MACE (HR: 0.89, 95\% CI: 0.83-0.96, P= 0.002) \& "CV death or hHF" composite (HR: 0.76, 95\% CI: 0.67-0.87, P= 0.000) demonstrated superiority. (Fig 1)

Although the present results seem to suggest benefit related to the "pump" and not the "pipe" in the Asian cohort, it is practically impossible to come to a definitive conclusion in view of the small number of patients \& events analysed. Another limitation of this analysis was the use of the term "Asian". This broad term encompasses several distinct ethnic identities and hence such generalization might not be appropriate.

This meta analysis indicates that the beneficial aspect of SGLT2i on "Asians" is limited to reduction in "hHF and CV death" but not in atherosclerotic events. However a focused CVOT on various Asian sub populations is the need of the hour. 

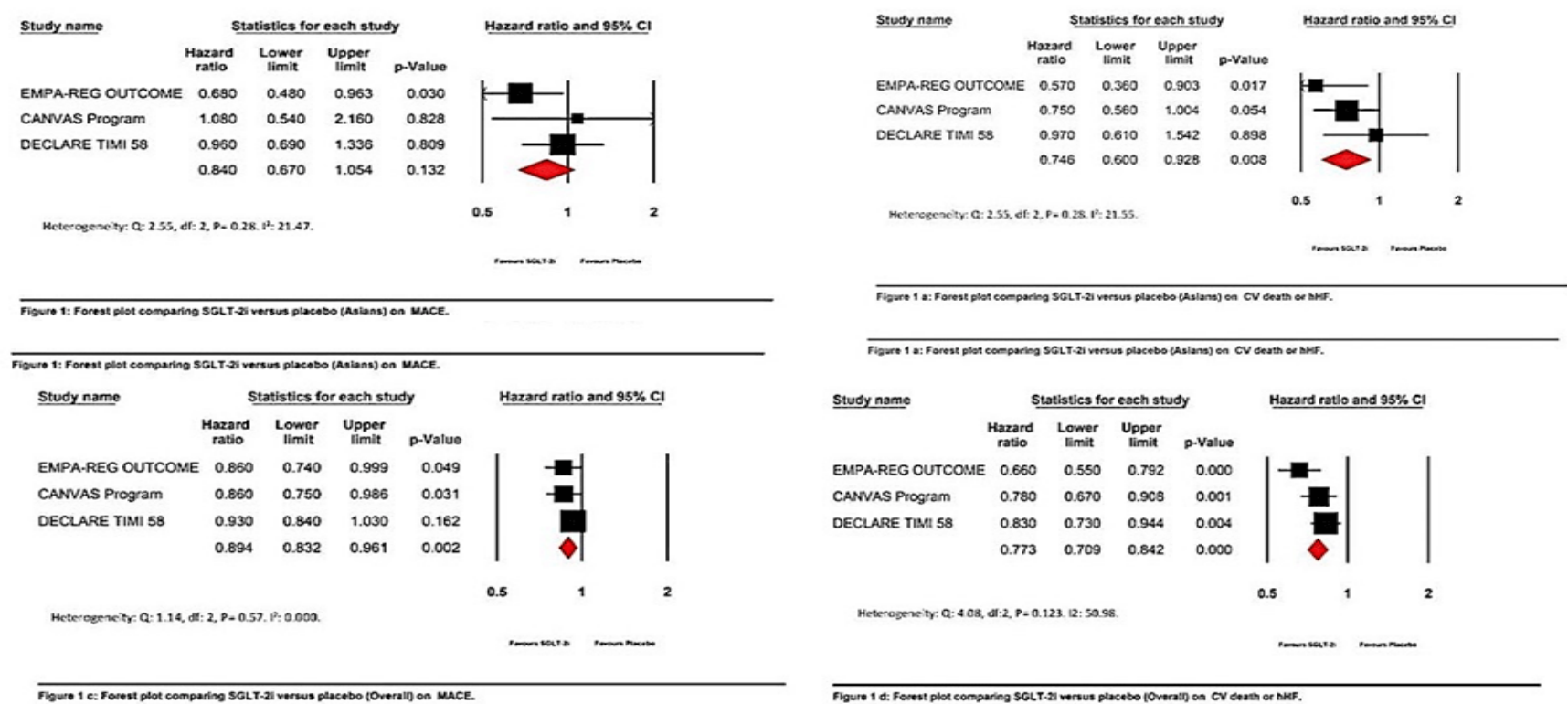

Figure 1: MACE-Asians; Fig 1 a: CV death or hHF- Asians; Fig 1 b: MACE-Overall; Fig 1 c: CV death or hHF-Overall. EMPA-REG OUTCOME: Empagliflozin

CANVAS Program: Canagliflozin

DECLARE TIMI 58: Dapagliflozin.

\section{References}

1. Verma S, Juni P, Mazer CD. Pumps, pipes and filter: Do SGLT-2 inhibitors cover it all? The Lancet. 2018; S0140-6736(18):32824-32831. Doi: 10.1016/S0140-6736(18)32824-1

2. Zinman B, Wanner C, Lachin JM, Fitchett D, Bluhmki E, Hantel S, et al. Empagliflozin, Cardiovascular Outcomes, and Mortality in Type 2 Diabetes. N Engl J Med 2015;373:2117-2128. Doi: 10.1056/NEJMoa1504720
3. Neal B, Perkovic V, Mahaffey KW, de Zeeuw D, Fulcher G, Erondu N, et al. Canagliflozin and Cardiovascular and Renal Events in Type 2 Diabetes. N Engl J Med 2017;377:644-657. Doi: 10.1056/NEJMoa1611925

4. Wiviott SD, Raz I, Bonaca MP, Mosenzon O, Kato ET, Cahn A, et al. Dapagliflozin and Cardiovascular Outcomes in type 2 Diabetes. NEJM. 2018. Doi: 10.1056/NEJMoa1812389 